\title{
Regulation of TDP-43 phosphorylation in aging and disease
}

\author{
Randall J. Eck • Brian C. Kraemer • \\ Nicole F. Liachko
}

Received: 4 February 2021 / Accepted: 6 May 2021 / Published online: 25 May 2021

(C) This is a U.S. government work and not under copyright protection in the U.S.; foreign copyright protection may apply 2021

\begin{abstract}
Insoluble inclusions of phosphorylated TDP-43 occur in disease-affected neurons of most patients with amyotrophic lateral sclerosis (ALS) and about half of patients with frontotemporal lobar degeneration (FTLD-TDP). Phosphorylated TDP-43 potentiates a number of neurotoxic effects including reduced liquid-liquid phase separation dynamicity, changes in splicing, cytoplasmic mislocalization, and aggregation. Accumulating evidence suggests a balance of kinase and phosphatase activities control TDP-43 phosphorylation. Dysregulation of these processes may lead to an increase in phosphorylated
\end{abstract}

\section{R. J. Eck · B. C. Kraemer}

Neuroscience Graduate Program, University

of Washington, Seattle, WA 98195, USA

R. J. Eck · B. C. Kraemer · N. F. Liachko $(\bowtie)$

Geriatric Research Education and Clinical Center, Seattle

Veterans Affairs Puget Sound Health Care System, 1660

South Columbian Way, Seattle, WA 98108, USA

e-mail: nliachko@uw.edu

B. C. Kraemer · N. F. Liachko

Division of Gerontology and Geriatric Medicine, Department of Medicine, University of Washington, Seattle, WA 98104, USA

B. C. Kraemer

Department of Psychiatry and Behavioral Sciences, University of Washington, Seattle, WA 98195, USA

B. C. Kraemer

Department of Laboratory Medicine \& Pathology, University of Washington, Seattle, WA 98104, USA
TDP-43, ultimately contributing to neurotoxicity and neurodegeneration in disease. Here we summarize the evolving understanding of major regulators of TDP-43 phosphorylation as well as downstream consequences of their activities. Interventions restoring kinase and phosphatase balance may be a generalizable therapeutic strategy for all TDP-43 proteinopathies including ALS and FTLD-TDP.

Keywords TDP-43 - Amyotrophic lateral sclerosis (ALS) · Frontotemporal lobar degeneration (FTLD) . Phosphorylation $\cdot$ Kinases $\cdot$ Phosphatases

\section{Introduction}

The TAR DNA-binding protein (TDP-43), encoded by the highly conserved TARDBP gene, is a nucleic acid binding protein originally identified as a transcriptional repressor of the human immunodeficiency virus type 1 (HIV-1) trans-activator response (TAR) long terminal repeats [1]. Since then, TDP-43 has been shown to participate in a variety of critical RNA metabolism activities including transcriptional regulation, pre-mRNA splicing of most transcripts, alternative splice site selection, mRNA stability and transport, and microRNA biogenesis (reviewed in [2]). The discovery that TDP-43 comprised the major protein component of ubiquitinated inclusions in both amyotrophic lateral sclerosis (ALS) and frontotemporal lobar degeneration (FTLD-TDP) began serious 
investigation into the role of TDP-43 in neurodegenerative diseases of aging [3-5]. Mutations identified within the TARDBP gene cause some familial cases of ALS, indicating pathological TDP-43 is not only a hallmark of disease, but also a driver of disease [6].

Pathological TDP-43 accumulates in the diseaseaffected neurons of $29 \%$ of ALS patients and $50 \%$ of FLTD patients [7-9]. This typically manifests as cytoplasmic deposition of C-terminally phosphorylated TDP-43 (Fig. 1A). The best characterized of these phosphorylation events occurs at serines 409 and 410 (S409/410), which are consistently phosphorylated in disease. In addition to being a robust and consistent pathological marker for TDP-43-positive inclusions [10], phosphorylated TDP-43 manifests in other neurodegenerative disease and aging [11]. TDP-43 pathology has been strongly associated with hippocampal sclerosis $[12,13]$ and accumulates in a subset of Alzheimer's disease cases, with dementia severity correlating dose dependently with phosphorylated TDP-43 deposition $[14,15]$. Furthermore, TDP-43 is increasingly recognized as a secondary pathology of other neurodegenerative disorders including Lewy body-related diseases and Huntington's disease [16, 17].

TDP-43 pathology can accumulate in nondemented aged individuals [18], although a recently classified TDP-43 proteinopathy, limbic predominant age-related TDP-43 proteinopathy (LATE), mimics Alzheimer's disease clinically in patients of advanced age. Compared to Alzheimer's disease, LATE exhibits a restricted distribution of TDP-43 pathology frequently associated with hippocampal sclerosis and does not display the plaques and tangles defining Alzheimer's disease [19]. Phosphorylated TDP-43 can also become deposited due to environmental insults as occurs in chronic traumatic encephalopathy (CTE) following brain injury [20]. The genetic or environmental causes of TDP-43 pathological deposition are unknown in the majority of patients with ALS, FTLD-TDP, Alzheimer's disease, Lewy body disease, Huntington's disease, or LATE. It remains possible that diverse mechanisms lead to the pathognomonic phenotypes observed in these different neurodegenerative diseases. Taken together, these observations support a role for TDP-43 in pathological aging and suggest pathological TDP-43 has a widespread impact on the cognitive decline associated with aging (reviewed in [21]).

\section{TDP-43 structure and function}

TDP-43 contains protein domains consistent with its known functions, including an N-terminus with a nuclear localization signal (NLS), two RNA recognition motifs (RRM1 and RRM2), and a C-terminus glycine-rich low-complexity domain (Fig. 1) [22]. RRM2 also contains a putative nuclear export signal, although it is not required or sufficient to mediate nuclear egress [23]. The N-terminus of TDP-43 functions in dimerization and can bind single-stranded DNA [24-26]. Both RRM1 and RRM2 are required for TDP-43 to bind UG-rich RNA or TG-rich DNA sequences, although RRM2 appears to play a supporting role to RRM1 [27-30]. Mutations that disrupt TDP-43 RNA binding may contribute to pathogenic mislocalization and aggregation in ALS and FTLDTDP [31]. While primarily localized in the nucleus, TDP-43 can freely shuttle between the nucleus and cytoplasm and has also been observed in neuronal mitochondria in ALS and FTLD-TDP [32, 33]. During cellular stress, TDP-43 localizes to the cytoplasm and can associate with stress granules through its RRM and C-terminus domains [34]. TDP-43 undergoes liquid-liquid phase separation, mediated by its C-terminus low-complexity domain [35, 36]. Deletion of this region prevents aggregation [37], and ALS-associated mutations in the C-terminus disrupt phase separation and promote aggregation [36, 38, 39]. Additionally, TDP-43 interactions with other proteins including ubiquilin-2 are mediated by the C-terminus domain [40-42]. Finally, through these various protein domains (Fig. 1), the cellular activities of TDP-43 are regulated by a combination of post-translational modifications, subcellular localization, and specific interactions with co-factors.

\section{TDP-43 phosphorylation sites in disease}

In ALS and FTLD-TDP, aggregated TDP-43 exhibits several post-translational modifications including ubiquitination, acetylation, SUMOylation, and phosphorylation [3, 4, 43, 44]. Of these modifications, abnormal phosphorylation of TDP-43 is a highly consistent marker of disease and is used diagnostically to identify TDP-43-positive protein inclusions in brain and spinal cord [5, 10, 45]. TDP-43 has 64 potential phosphorylation sites: 41 serine (Ser), 15 threonine 
A

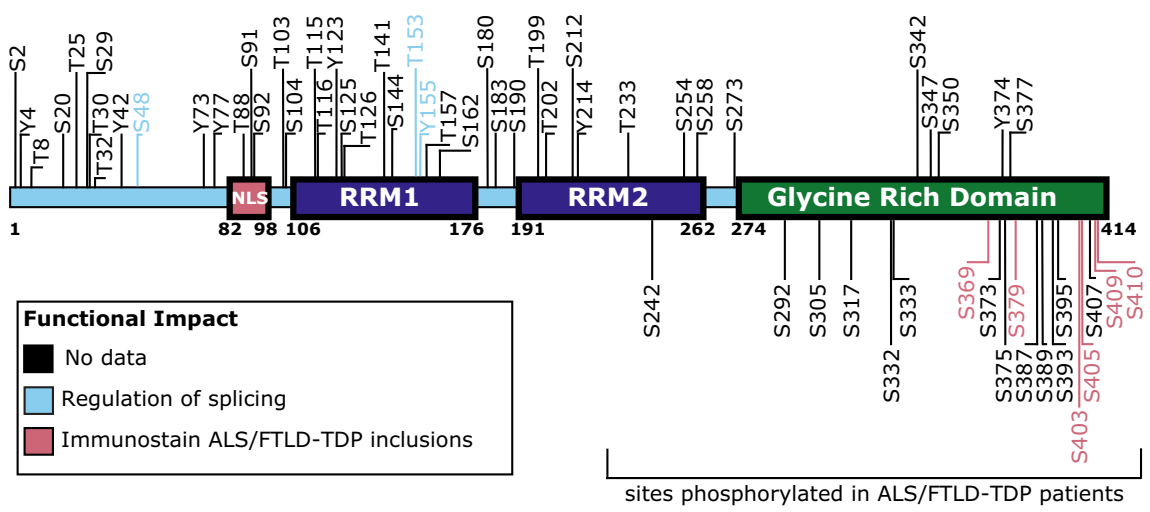

B

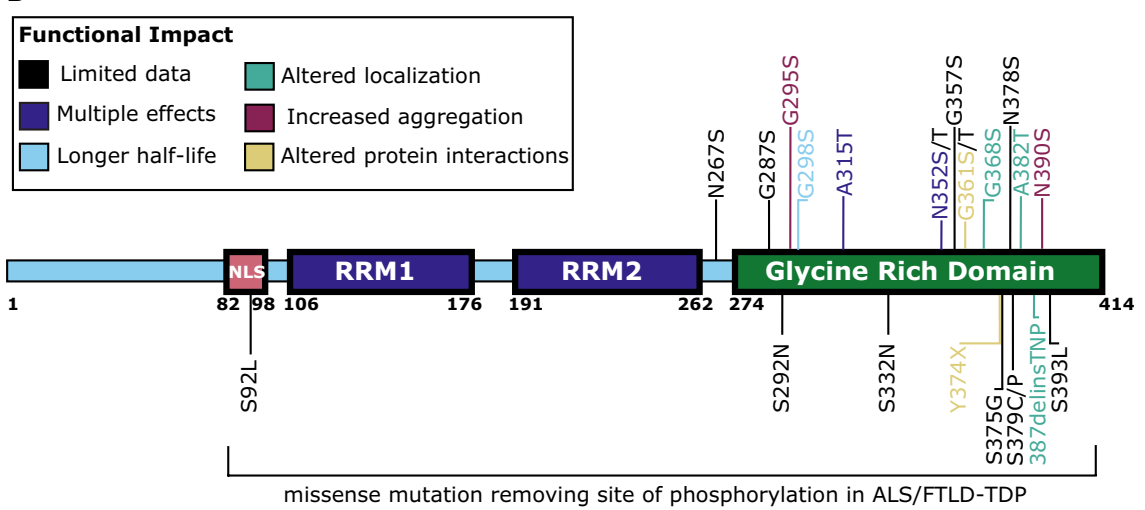

Fig. 1 TDP-43 protein domains and phosphorylation sites. A TDP-43 is a 414 amino acid nucleic acid binding protein involved in RNA metabolism activities including transcriptional regulation, pre-mRNA splicing, mRNA stability and transport, and microRNA biogenesis. TDP-43 contains an $\mathrm{N}$-terminus with a nuclear localization signal (NLS), two RNA recognition motifs (RRM1 and RRM2), and a C-terminus glycine-rich low-complexity domain. TDP-43 has 64 potential phosphorylation sites: 41 serine (Ser), 15 threonine (Thr), and 8 tyrosine (Tyr) residues. A subset of these sites (shown below the TDP-43 diagram) have been identified phosphorylated in ALS and FTLD-TDP patients. Many of TDP-43's phosphorylation sites have not been fully characterized although in vivo and in vitro studies have used immunostaining to demonstrate

(Thr), and 8 tyrosine (Tyr) residues (Fig. 1A). A subset of these sites become phosphorylated in vivo, a smaller group have been observed by IHC in ALS and FTLD-TDP patients, and fewer have been functionally characterized. Mass spectrometry of insoluble TDP-43 from the brain tissue of two ALS patients showed TDP-43 was phosphorylated at 17 sites, 16 of which reside in the glycine-rich $\mathrm{C}$-terminus domain [46]. In addition to these 17 sites, two other sites S369 and S410 — are known to be phosphorylated in their presence in TDP-43-positive inclusions (red text), or have linked specific sites to the regulation of TDP-43 splicing activity (blue text) (see inset box). B A subset of disease-causing TDP-43 missense mutations delete (shown below the TDP43 diagram) or introduce (shown above the TDP-43 diagram) phosphorylation sites. Some of these mutations' impact on TDP-43 pathology have been characterized, as represented by their font color (see inset box and color coding of text), including increased TDP-43 cytoplasmic mislocalization, altered protein-protein interactions, increased aggregation, and/or increased protein half-life. While additional characterization is needed, these mutations suggest a key role for the regulation of phosphorylation in TDP-43 function and disease

ALS and FTLD-TDP patients [5, 10, 47, 48]. Of these 19 sites, phosphorylation at S369, S379, S403/404, and $\mathbf{S} 409 / 410$ consistently occurs in the context of disease suggesting a potential pathological role (Fig. 1A). The distribution of the remaining 13 sites in disease has not yet been corroborated with IHC or other independent means, due to a lack of phosphorylation site-specific antibodies. Some of the identified phosphorylation events may represent the consequences of priming by disease-linked sites. 
At least twenty disease-linked missense mutations have been identified that introduce or delete phosphorylation sites potentially altering TDP-43's localization, aggregation, half-life, or protein-protein interactions (Fig. 1B) (reviewed in [6]). These mutations may also promote phosphorylation at nearby phospho-sites. However, characterization of the biological impact of these changes is ongoing. It remains unknown whether TDP-43 phosphorylation status changes with age to predict disease onset, or whether there are disease-specific patterns of TDP-43 phosphorylation. However, derangement of kinase or phosphatase activities with aging or disease may promote increased TDP-43 phosphorylation leading to dysfunction and eventual neurodegeneration. Given the ubiquitous presence of TDP-43 phosphorylation in disease, understanding the functional impact of specific phosphorylation sites is likely to provide important insights into disease mechanisms.

\section{Regulation of TDP-43 phosphorylation}

While phosphorylation of TDP-43 could represent an aberrant cellular process during disease, several lines of evidence suggest this is a regulated event. To date, five kinases have been shown to directly phosphorylate TDP-43: casein kinases 1 and 2 (CK1 and CK2), cell division cycle 7 (CDC7), and tau tubulin kinases 1 and 2 (TTBK1 and TTBK2) (Fig. 2) [5, 49-51]. In vitro, the kinases CK1 and CK2 promote TDP-43 polymerization into electron microscopy-evaluated structures reminiscent of those from FTLD-TDP [52], and all five kinases can phosphorylate purified TDP-43, with CK1 phosphorylating TDP-43 at 29 known sites [5, 49-51]. These kinases actively target TDP-43 in vivo, as modulation of kinase activity alters TDP-43 phosphorylation. For example, inhibitors of the kinases CK1 and CDC7 have been shown to prevent accumulation of phosphorylated TDP-43 and protect against neuron loss, while overexpression of CDC7, TTBK1, or TTBK2 promotes TDP-43 phosphorylation [49, 53-57]. CDC7, TTBK1, and TTBK2 also co-localize with aggregated, phosphorylated TDP43 in ALS and FTLD-TDP [49, 50]. Additionally, casein kinase $1 \varepsilon(C S N K 1 E)$ mRNA levels correlate with phosphorylated TDP-43 in ALS patient tissue and overexpression of CSNK1E in iPSC-derived

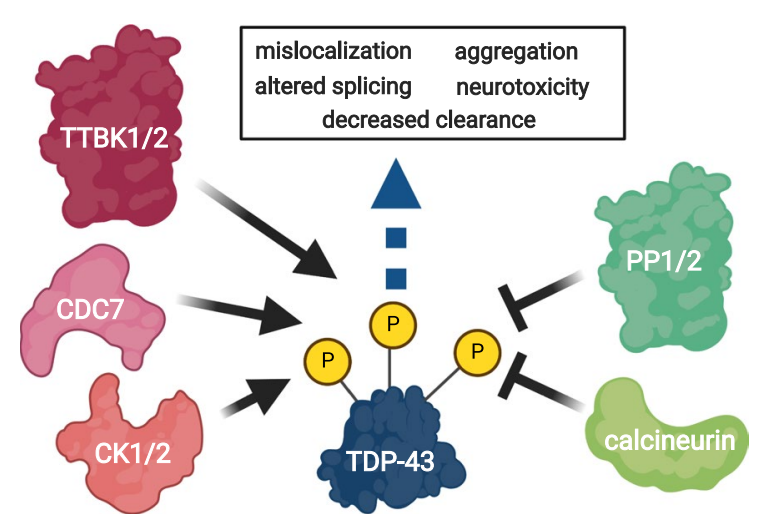

Fig. 2 Regulation and consequences of TDP-43 phosphorylation. TDP-43 phosphorylation is linked to deleterious functional changes including altered splicing activity, cellular mislocalization, decreased turnover, changes in solubility, and increased neurotoxicity. Five kinases have been shown to directly phosphorylate TDP-43: casein kinases 1 and 2 (CK1 and CK2), cell division cycle 7 (CDC7), and tau tubulin kinases 1 and 2 (TTBK1 and TTBK2). The phosphatases PP1, PP2, and calcineurin have been found to interact with and dephosphorylate TDP-43. Kinase and phosphatase activities regulate TDP-43 phosphorylation in what is likely to be dynamic cellular process with potential impacts on TDP-43 activities, interaction partners, and localization. Interventions restoring kinase and phosphatase balance may be a generalizable therapeutic strategy for all TDP-43 proteinopathies including ALS and FTLD-TDP

motor neuron lines promotes TDP-43 cytoplasmic accumulation [58]. Finally, although not shown to directly phosphorylate TDP-43, the kinase MEK promotes increased dual phosphorylation at $\mathrm{T} 153 / \mathrm{Y} 155$ in the TDP-43 RRM1 domain and nucleolar localization of TDP-43 during the heat shock response in SH-SY5Y cells [59]. It remains unknown whether TDP-43 kinases act in a defined temporal sequence, or whether their activities are coordinated with C-terminal truncation of TDP-43. The full phosphorylation profiles and site-preferences of these kinases on TDP-43 have also yet to be fully characterized. It is clear, though, that phosphorylation of TDP-43 by these kinases modifies pathology in model systems, and likely serves normal endogenous roles in regulating protein activity in conjunction with phosphatases.

Phosphatases catalyze the reverse reaction, removing phosphate groups from modified tyrosine, serine, and threonine residues. The phosphatases PP1, PP2, and calcineurin have been found to dephosphorylate TDP-43 (Fig. 2). All three 
phosphatases can physically interact with TDP-43, as PP1 and PP2 co-immunoprecipitated with TDP43 from HEK-293FT cells, while calcineurin interacted with the C-terminus of TDP-43 in a yeast2-hybrid assay [60, 61]. In addition, calcineurin and TDP-43 co-immunoprecipitated from human brain lysate [62]. PP1 can dephosphorylate purified human TDP-43 at pathological sites S379, S403, S404, S409, and S410 in vitro, while calcineurin has been shown to dephosphorylate TDP-43 at S409 and S410 [60, 61]. In mammalian cell culture, pharmacological inhibition of PP1 using the drug Calyculin A, or inhibition of calcineurin using the drugs FK506 or Cyclosporin A, drives accumulation of phosphorylated TDP-43 [60, 61]. In TDP43-expressing transgenic C. elegans, genetic loss of the sole calcineurin A homolog, tax- 6 , dramatically worsens motor phenotypes, phosphorylated TDP-43 accumulation, and neurodegeneration. Calcineurin and phosphorylated TDP-43-positive lesions co-localize in cortical neurons from patients with FTLD-TDP, and in spinal cord motor neurons from patients with ALS, suggesting calcineurin may be recruited to dephosphorylate TDP-43 in human disease [61]. Calcineurin activity is decreased in both sporadic and familial ALS patients, making calcineurin dysregulation a possible contributor to disease $[63,64]$. Taken together, kinase and phosphatase activities regulate TDP-43 phosphorylation in what is likely to be a dynamic cellular process influenced by aging or disease and with consequences for TDP-43 activities, interaction partners, and localization.

\section{Consequences of TDP-43 phosphorylation}

While there has not yet been an exhaustive survey of the functional consequences of TDP-43 phosphorylation at all possible phospho-sites, several sites have been examined in detail in model systems.

Regulation of splicing activity

In vitro and in vivo models have found phosphorylation at S48 and T153/Y155 can alter TDP-43's splicing activity [59, 65]. A phosphomimetic substitution at S48 (S48E) reduces TDP-43's alternative splicing repressor activity, resulting in a $40 \%$ reduction in the inclusion of exon 9 in the mRNA of cystic fibrosis transmembrane conductance regulator (CFTR) of HeLa cells [65]. Mutations T153E/Y155E, a likely dual phosphomimic, similarly reduce TDP-43 splicing activity, decreasing the inclusion of CFTR exon 9 by $30 \%$ compared to wild type. T153E/Y155E also decreases TDP-43 A(GU) 6 RNA binding affinity fivefold, while not altering solubility or degradation [59], further suggesting a specific role for phosphorylation in regulating splicing activity.

\section{Subcellular localization}

In addition to altered splicing activity, TDP-43 phosphorylation at T153/Y155 in SH-SY5Y cells regulates TDP-43 recruitment to nucleoli [59]. TDP-43 can also accumulate in mitochondria, and a phosphomimetic G298D mutation within one of TDP-43's mitochondrial localization signals increases TDP-43 mitochondrial localization in HEK-293 cells compared to wild type [32]. TDP-43 is predominantly located in the nucleus; however, in a model of intercranial hemorrhage $(\mathrm{ICH})$ in rats and rat primary neurons, TDP-43 translocates to the cytoplasm following ICH. Overexpressed, non-phosphorylatable TDP-43 mutants S409A/S410A remain in the nucleus following $\mathrm{ICH}$, suggesting a role for phosphorylation in TDP-43 cytoplasmic localization. The authors also found TDP-43 S409A/S410A mutations correlated with a reduction in markers of autophagosome-lysosome fusion and increased mTOR and dynactin1 levels following $\mathrm{ICH}$, potentially due to the maintenance of nuclear localized TDP-43 [66]. Following ER stress, the kinase CK1 promotes TDP-43 cytoplasmic accumulation in NSC-34 cells [67]. Expression of phosphomimetic mutations S375E and S387E/ S389E/S393E/S395E in TDP-43's low-complexity domain also significantly increases TDP-43 cytoplasmic localization compared to wild type in HeLa cells [68]. In other models, cytoplasmic accumulation of TDP-43 is sufficient to induce aggregation and phosphorylation. For example, the deletion of TDP-43's NLS induces TDP-43 aggregation in phase separated droplets in SH-SY5Y cells. Long-lived TDP-43 phase separated droplets also recruit phosphorylated TDP43 [69]. In transgenic mice, expression of human TDP-43 with a defective NLS results in accumulation of cytoplasmic phosphorylated insoluble TDP-43, 
accompanied by motor impairment, neurodegeneration, and rapid death [70].

Solubility and aggregate formation

Independent of localization, phosphorylation of TDP43 has been linked to changes in solubility and aggregate formation. A phosphomimetic substitution at S48 (S48E) dramatically reduces the ability of TDP43 to polymerize. Furthermore, S48E slows TDP-43 phase separation and results in more soluble phase separated TDP-43 nuclear assembles [65]. A recent study also found this mutation disrupted the formation of anisosomes, distinct phase separated nuclear assemblies formed when TDP-43 polymerizes following an inhibition of RNA binding [71]. Beyond phase separation, phosphorylation at S409/410 in the C-terminus of TDP-43 fragments increases their resistance to proteasomal degradation [72]. Phosphorylation of TDP-43 at S403/404 in HeLa cells by CK1, CK2, TTBK1, or TTBK2 inhibits calpain-dependent cleavage of TDP-43 compared to non-phosphorylated TDP-43 in vitro [73]. Previous research found calpain cleaved TDP-43 fragments are more soluble and suggests preventing calpain-dependent digestion could increase TDP-43 aggregation propensity [74]. In another experiment, the co-expression of constitutively active mutant CSNK1E and TDP-43 in SH-SY5Y cells resulted in dual phosphorylation of TDP-43 at S393/395 and increased TDP-43 aggregation. Mutation of S393/395 to the non-phosphorylatable S393A/S395A reduces the levels of TDP-43 phosphorylation at other sites including S409/410, as well as the total amount of accumulated TDP-43 in the sarksoyl-insoluble fraction of cells [75]. Another study suggests phosphorylation at S409/410 is a secondary event in seed-dependent TDP-43 aggregation [76]. In N2A or COS-7 cells expressing $15 \mathrm{kDa}$ and $25 \mathrm{kDa}$ C-terminus fragments of TDP-43, phosphorylation of $\mathrm{S} 409 / 410$ is significantly more common in insoluble fragments further supporting a role for S409/410 phosphorylation in reducing solubility [76].

However, not all studies suggest phosphorylation promotes aggregation. In HEK-293 cells, TDP$4315 \mathrm{kDa}$ and $25 \mathrm{kDa}$ fragments with phosphomimetic mutations S409D/S410D show 60\% less aggregation compared to wild type fragments suggesting a possible compensatory role for TDP-43 phosphorylation. Non-phosphorylatable mutations S409A/S410A also reduce aggregation, but only by $15 \%$ [77]. Likewise, expressing TDP-43 C-terminus fragments with S403A/S404A or S409A/ S410A non-phosphorylatable mutations in Drosophila does not alter TDP-43 aggregation. Various combinations of phosphomimetic mutations S379E, S403E, S404E, S409E, and S410E, or expression of the kinase $\mathrm{CK} 2 \alpha$, are able to reduce the number of aggregates in cells. In these studies, S409/410 phosphorylation occurs after aggregation suggesting TDP-43 phosphorylation may be compensatory, delaying the formation of larger aggregates by electrostatic repulsion at the expense of decreasing TDP-43 turnover [78].

Timing of phosphorylation

While the timing and subcellular localization of TDP-43 phosphorylation in disease remains largely unexplored, two recent studies support S409/410 phosphorylation occurring after aggregate formation. To drive aggregation, an optogenetic TDP43 construct that undergoes oligomerization after blue light exposure (optoTDP43) was expressed in HEK-293 cells or ReNcell cortical neuron cultures. After induction, optoTDP43 forms stable neurotoxic inclusions, a subset of which are phosphorylated [79]. Another study induced the formation of stress granules using optogenetic multimerization of the stress granule scaffold protein G3BP1. TDP43 is recruited to these OptoGranules and became immunopositive for $\mathrm{S} 409 / 410$ phosphorylation after recruitment [80]. During transient stress, TDP-43 recruitment to stress granules inhibits TDP-43 phosphorylation at $\mathrm{S} 409 / 410$. It is possible the stress granule environment excludes stress-responsive kinases, altering TDP-43 interactions with kinases and phosphatases and delaying cytoplasmic accumulation of TDP-43 [81].

\section{Neurotoxicity}

In C. elegans models, neuronal expression of human TDP-43 with fALS mutations G290A or M337V kills motor neurons. Prevention of S409/410 phosphorylation with non-phosphorylatable mutations S409A/S410A improves locomotor dysfunction 
[82]. Similarly, co-expression of mutant human TDP-43 Q331K with doubletime, the fly homolog of CSNK1E, in the Drosophila eye increases S409/410 phosphorylation and the formation of toxic oligomeric species. Cytotoxicity of TDP-43 oligomers is also enhanced in SY5Y cells when treated with a recombinant rat CK1 [83].

\section{Summary}

Taken together, the referenced studies demonstrate that TDP-43 phosphorylation influences normal TDP-43 functions including RNA binding, alternative splicing, localization, phase separation, aggregation, and clearance. Yet, many questions remain with regard to the sub-cellular localization, timing in disease progression, potential for additional phosphorylation priming, and full functional impact of site-specific phosphorylation. For example, phospho-specific antibodies raised against TDP-43 phosphorylated at S369 and S379 immunostain insoluble cytoplasmic inclusions in ALS/FTLD-TDP patient brain tissue, but the functional impact of phosphorylation at these sites on TDP-43 is unknown. Disease-causing missense mutations introducing or deleting TDP-43 phosphorylation sites also point to important roles for phosphorylation in health and disease (Fig. 1B). It remains unknown whether changes in the pattern or degree of TDP-43 phosphorylation presage development of neuronal dysfunction or neurodegeneration, or whether normal aging affects regulation of TDP-43 phosphorylation. Nevertheless, this work represents an important start to understanding the variety of regulatory effects of TDP-43 phosphorylation.

The kinases and phosphatases that regulate TDP43 phosphorylation are possible therapeutic targets in TDP-43 proteinopathies. However, a single primary TDP-43 kinase has not been determined in neurons; given possible overlap or redundancy in TDP-43 kinases' activities, effective treatments may require multiple small molecule inhibitors targeting several TDP-43 kinases to fully prevent TDP-43 phosphorylation. Similarly, phosphatase activation could be used to drive clearance of phosphorylated TDP-43, although given the many known targets of calcineurin, PP1, and PP2, this strategy would need to be carefully evaluated to screen for potential adverse effects. Another strategy could be targeted clearance of phosphorylated TDP-43, potentially using an immunotherapy approach, or by enhancing endogenous cellular clearance mechanisms such as autophagy. Given the interplay among TDP-43 phosphorylation, aggregation, and cellular localization, treatments focused on disaggregation or restoration of nuclear localization could reduce TDP-43 phosphorylation as a downstream consequence, with potential neuroprotective benefits. There is an urgent need for effective therapies for TDP-43 proteinopathies; modifying TDP-43 phosphorylation holds both promise and challenge for ALS and FTLD-TDP.

Acknowledgements We thank the reviewers for helpful comments and suggestions. We thank the researchers who have contributed their efforts to this exciting emerging area, some of whom we were able to highlight here. Figure 2 was created with BioRender.com.

Funding This work was supported by grants from the Department of Veterans Affairs [Merit Review Grants \#I01BX002619 to B.C.K. and \#I01BX004044 to N.F.L.] and National Institutes of Health [R01 NS064131 to B.C.K].

\section{Declarations}

Conflict of interest The authors declare no competing interests.

Open Access This article is licensed under a Creative Commons Attribution 4.0 International License, which permits use, sharing, adaptation, distribution and reproduction in any medium or format, as long as you give appropriate credit to the original author(s) and the source, provide a link to the Creative Commons licence, and indicate if changes were made. The images or other third party material in this article are included in the article's Creative Commons licence, unless indicated otherwise in a credit line to the material. If material is not included in the article's Creative Commons licence and your intended use is not permitted by statutory regulation or exceeds the permitted use, you will need to obtain permission directly from the copyright holder. To view a copy of this licence, visit http://creativecommons.org/licenses/by/4.0/.

\section{References}

1. Ou SH, et al. Cloning and characterization of a novel cellular protein, TDP-43, that binds to human immunodeficiency virus type 1 TAR DNA sequence motifs. J Virol. 1995;69(6):3584-96.

2. Guerrero EN, et al. TDP-43/FUS in motor neuron disease: complexity and challenges. Prog Neurobiol. 2016;145-146:78-97. 
3. Neumann M, et al. Ubiquitinated TDP-43 in frontotemporal lobar degeneration and amyotrophic lateral sclerosis. Science. 2006;314(5796):130-3.

4. Arai T, et al. TDP-43 is a component of ubiquitin-positive tau-negative inclusions in frontotemporal lobar degeneration and amyotrophic lateral sclerosis. Biochem Biophys Res Commun. 2006;351(3):602-11.

5. Hasegawa M, et al. Phosphorylated TDP-43 in frontotemporal lobar degeneration and amyotrophic lateral sclerosis. Ann Neurol. 2008;64(1):60-70.

6. Buratti E. Functional significance of TDP-43 mutations in disease. Adv Genet. 2015;91:1-53.

7. Mackenzie IR. The neuropathology of FTD associated With ALS. Alzheimer Dis Assoc Disord. 2007;21(4):S44-9.

8. Kawakami I, Arai T, Hasegawa M. The basis of clinicopathological heterogeneity in TDP-43 proteinopathy. Acta Neuropathol. 2019;138(5):751-70.

9. Mann DMA, Snowden JS. Frontotemporal lobar degeneration: Pathogenesis, pathology and pathways to phenotype. Brain Pathol. 2017;27(6):723-36.

10. Neumann M, et al. Phosphorylation of $S 409 / 410$ of TDP-43 is a consistent feature in all sporadic and familial forms of TDP-43 proteinopathies. Acta Neuropathol. 2009;117(2):137-49.

11. McAleese KE, et al. TDP-43 pathology in Alzheimer's disease, dementia with Lewy bodies and ageing. Brain Pathol. 2017;27(4):472-9.

12. Nag S, et al. Hippocampal sclerosis and TDP-43 pathology in aging and Alzheimer disease. Ann Neurol. 2015;77(6):942-52.

13. Amador-Ortiz C, et al. TDP-43 immunoreactivity in hippocampal sclerosis and Alzheimer's disease. Ann Neurol. 2007;61(5):435-45.

14. James BD, et al. TDP-43 stage, mixed pathologies, and clinical Alzheimer's-type dementia. Brain. 2016;139(11):2983-93.

15. Uryu $\mathrm{K}$, et al. Concomitant TAR-DNA-binding protein 43 pathology is present in Alzheimer disease and corticobasal degeneration but not in other tauopathies. J Neuropathol Exp Neurol. 2008;67(6):555-64.

16. Nakashima-Yasuda H, et al. Co-morbidity of TDP-43 proteinopathy in Lewy body related diseases. Acta Neuropathol. 2007;114(3):221-9.

17. Schwab C, et al. Colocalization of transactivation-responsive DNA-binding protein 43 and huntingtin in inclusions of Huntington disease. J Neuropathol Exp Neurol. 2008;67(12):1159-65.

18. Kovacs GG, et al. Non-Alzheimer neurodegenerative pathologies and their combinations are more frequent than commonly believed in the elderly brain: a community-based autopsy series. Acta Neuropathol. 2013;126(3):365-84.

19. Nelson PT, et al. Limbic-predominant age-related TDP-43 encephalopathy (LATE): consensus working group report. Brain. 2019;142(6):1503-27.
20. McKee AC, et al. TDP-43 proteinopathy and motor neuron disease in chronic traumatic encephalopathy. J Neuropathol Exp Neurol. 2010;69(9):918-29.

21. Zhang L, et al. TDP-43 and limbic-predominant agerelated TDP-43 encephalopathy. Front Aging Neurosci. 2019;11:376.

22. Ayala YM, et al. Human, Drosophila, and C.elegans TDP43: nucleic acid binding properties and splicing regulatory function. J Mol Biol. 2005;348(3):575-88.

23. Pinarbasi ES, et al. Active nuclear import and passive nuclear export are the primary determinants of TDP-43 localization. Sci Rep. 2018;8(1):7083.

24. Chang CK, et al. The N-terminus of TDP-43 promotes its oligomerization and enhances DNA binding affinity. Biochem Biophys Res Commun. 2012;425(2):219-24.

25. Afroz T, et al. Functional and dynamic polymerization of the ALS-linked protein TDP-43 antagonizes its pathologic aggregation. Nat Commun. 2017;8(1):45.

26. Qin $\mathrm{H}$, et al. TDP-43 $\mathrm{N}$ terminus encodes a novel ubiquitin-like fold and its unfolded form in equilibrium that can be shifted by binding to ssDNA. Proc Natl Acad Sci U S A. 2014;111(52):18619-24.

27. Buratti E, Baralle FE. Characterization and functional implications of the RNA binding properties of nuclear factor TDP-43, a novel splicing regulator of CFTR exon 9. J Biol Chem. 2001;276(39):36337-43.

28. Kuo PH, et al. Structural insights into TDP-43 in nucleicacid binding and domain interactions. Nucleic Acids Res. 2009;37(6):1799-808.

29. Kuo PH, et al. The crystal structure of TDP-43 RRM1DNA complex reveals the specific recognition for UG- and TG-rich nucleic acids. Nucleic Acids Res. 2014;42(7):4712-22.

30. Furukawa Y, et al. A molecular mechanism realizing sequence-specific recognition of nucleic acids by TDP-43. Sci Rep. 2016;6:20576.

31. Chen HJ, et al. RRM adjacent TARDBP mutations disrupt RNA binding and enhance TDP-43 proteinopathy. Brain. 2019;142(12):3753-70.

32. Wang W, et al. The inhibition of TDP-43 mitochondrial localization blocks its neuronal toxicity. Nat Med. 2016;22(8):869-78.

33. Ayala YM, et al. Structural determinants of the cellular localization and shuttling of TDP-43. J Cell Sci. 2008;121(Pt 22):3778-85.

34. Colombrita C, et al. TDP-43 is recruited to stress granules in conditions of oxidative insult. $\mathrm{J}$ Neurochem. 2009;111(4):1051-61.

35. Conicella AE, et al. TDP-43 alpha-helical structure tunes liquid-liquid phase separation and function. Proc Natl Acad Sci U S A. 2020;117(11):5883-94.

36. Conicella AE, et al. ALS Mutations disrupt phase separation mediated by alpha-helical structure in the TDP43 low-complexity C-terminal domain. Structure. 2016;24(9):1537-49.

37. Johnson BS, et al. TDP-43 is intrinsically aggregation-prone and ALS-linked mutations 
accelerate aggregation and increase toxicity. J Biol Chem. 2009;284(30):20329-39.

38. Lim L, et al. ALS-causing mutations significantly perturb the self-assembly and interaction with nucleic acid of the intrinsically disordered prion-like domain of TDP-43. PLoS Biol. 2016;14(1):e1002338.

39. Schmidt HB, Rohatgi R. In vivo formation of vacuolated multi-phase compartments lacking membranes. Cell Rep. 2016;16(5):1228-36.

40. Fuentealba RA, et al. Interaction with polyglutamine aggregates reveals a Q/N-rich domain in TDP-43. J Biol Chem. 2010;285(34):26304-14.

41. Cassel JA, Reitz AB. Ubiquilin-2 (UBQLN2) binds with high affinity to the C-terminal region of TDP-43 and modulates TDP-43 levels in $\mathrm{H} 4$ cells: characterization of inhibition by nucleic acids and 4-aminoquinolines. Biochim Biophys Acta. 2013;1834(6):964-71.

42. Buratti E, Baralle FE. TDP-43: gumming up neurons through protein-protein and protein-RNA interactions. Trends Biochem Sci. 2012;37(6):237-47.

43. Cohen TJ, et al. An acetylation switch controls TDP43 function and aggregation propensity. Nat Commun. 2015;6:5845.

44. Seyfried NT, et al. Multiplex SILAC analysis of a cellular TDP-43 proteinopathy model reveals protein inclusions associated with SUMOylation and diverse polyubiquitin chains. Mol Cell Proteomics. 2010;9(4):705-18.

45. Inukai $\mathrm{Y}$, et al. Abnormal phosphorylation of Ser409/410 of TDP-43 in FTLD-U and ALS. FEBS Lett. 2008;582(19):2899-904.

46. Kametani F, et al. Mass spectrometric analysis of accumulated TDP-43 in amyotrophic lateral sclerosis brains. Sci Rep. 2016;6:23281.

47. Neumann M, et al. Antibody against TDP-43 phosphorylated at serine 375 suggests conformational differences of TDP-43 aggregates among FTLD-TDP subtypes. Acta Neuropathol. 2020;140(5):645-58.

48. Neumann M, et al. Correction to: Antibody against TDP43 phosphorylated at serine 369 suggests conformational differences of TDP-43 aggregates among FTLD-TDP subtypes. Acta Neuropathol. 2021;141(1):137.

49. Liachko NF, et al. CDC7 inhibition blocks pathological TDP-43 phosphorylation and neurodegeneration. Ann Neurol. 2013;74(1):39-52.

50. Liachko NF, et al. The tau tubulin kinases TTBK1/2 promote accumulation of pathological TDP-43. PLoS Genet. 2014;10(12):e1004803.

51. Kametani F, et al. Identification of casein kinase-1 phosphorylation sites on TDP-43. Biochem Biophys Res Commun. 2009;382(2):405-9.

52. Carlomagno Y, et al. Casein kinase II induced polymerization of soluble TDP-43 into filaments is inhibited by heat shock proteins. PLoS One. 2014;9(3):e90452.

53. Taylor LM, et al. Pathological phosphorylation of tau and TDP-43 by TTBK1 and TTBK2 drives neurodegeneration. Mol Neurodegener. 2018;13(1):7.
54. Salado IG, et al. Protein kinase CK-1 inhibitors as new potential drugs for amyotrophic lateral sclerosis. J Med Chem. 2014;57(6):2755-72.

55. Rojas-Prats E, et al. Targeting nuclear protein TDP-43 by cell division cycle kinase 7 inhibitors: A new therapeutic approach for amyotrophic lateral sclerosis. Eur J Med Chem. 2021;210:112968.

56. Martinez-Gonzalez L, et al. Motor neuron preservation and decrease of in vivo TDP-43 phosphorylation by protein CK-1delta kinase inhibitor treatment. Sci Rep. 2020;10(1):4449.

57. Alquezar C, et al. Targeting TDP-43 phosphorylation by Casein Kinase-1delta inhibitors: a novel strategy for the treatment of frontotemporal dementia. Mol Neurodegener. 2016;11(1):36.

58. Krach F, et al. Transcriptome-pathology correlation identifies interplay between TDP-43 and the expression of its kinase CK1E in sporadic ALS. Acta Neuropathol. 2018;136(3):405-23.

59. Li W, et al. Heat shock-induced phosphorylation of TAR DNA-binding protein 43 (TDP-43) by MAPK/ ERK kinase regulates TDP-43 function. J Biol Chem. 2017;292(12):5089-100.

60. Gu J, et al. Protein phosphatase 1 dephosphorylates TDP43 and suppresses its function in tau exon 10 inclusion. FEBS Lett. 2018;592(3):402-10.

61. Liachko NF, et al. The phosphatase calcineurin regulates pathological TDP-43 phosphorylation. Acta Neuropathol. 2016;132(4):545-61.

62. Davis SA, et al. TDP-43 expression influences amyloidbeta plaque deposition and tau aggregation. Neurobiol Dis. 2017;103:154-62.

63. Ferri A, et al. Activity of protein phosphatase calcineurin is decreased in sporadic and familial amyotrophic lateral sclerosispatients. J Neurochem. 2004;90(5):1237-42.

64. Wagey R, Krieger C, Shaw CA. Abnormal dephosphorylation effect on NMDA receptor regulation in ALS spinal cord. Neurobiol Dis. 1997;4(5):350-5.

65. Wang A, et al. A single N-terminal phosphomimic disrupts TDP-43 polymerization, phase separation, and RNA splicing. EMBO J. 2018;37(5):e97452.

66. Sun L, et al. TAR DNA binding protein-43 loss of function induced by phosphorylation at $\mathrm{S} 409 / 410$ blocks autophagic flux and participates in secondary brain injury after intracerebral hemorrhage. Front Cell Neurosci. 2018;12:79.

67. Hicks DA, et al. Endoplasmic reticulum stress signalling induces casein kinase 1-dependent formation of cytosolic TDP-43 inclusions in motor neuron-like cells. Neurochem Res. 2020;45(6):1354-64.

68. Newell K, et al. Dysregulation of TDP-43 intracellular localization and early onset ALS are associated with a TARDBP S375G variant. Brain Pathol. 2019;29(3):397-413.

69. Gasset-Rosa F, et al. Cytoplasmic TDP-43 De-mixing Independent of stress granules drives inhibition of nuclear 
import, loss of nuclear TDP-43, and cell death. Neuron. 2019;102(2):339-57 e7.

70. Walker AK, et al. Functional recovery in new mouse models of ALS/FTLD after clearance of pathological cytoplasmic TDP-43. Acta Neuropathol. 2015;130(5):643-60.

71. $\mathrm{Yu} \mathrm{H}$, et al. HSP70 chaperones RNA-free TDP-43 into anisotropic intranuclear liquid spherical shells. Science. 2021;371(6529):eabb4309.

72. Zhang YJ, et al. Phosphorylation regulates proteasomalmediated degradation and solubility of TAR DNA binding protein-43 C-terminal fragments. Mol Neurodegener. 2010;5:33.

73. Yamashita T, Teramoto S, Kwak S. Phosphorylated TDP43 becomes resistant to cleavage by calpain: A regulatory role for phosphorylation in TDP-43 pathology of ALS/ FTLD. Neurosci Res. 2016;107:63-9.

74. Yamashita T, et al. A role for calpain-dependent cleavage of TDP-43 in amyotrophic lateral sclerosis pathology. Nat Commun. 2012;3:1307.

75. Nonaka T, et al. Phosphorylation of TAR DNA-binding protein of $43 \mathrm{kDa}$ (TDP-43) by truncated casein kinase 1delta triggers mislocalization and accumulation of TDP43. J Biol Chem. 2016;291(11):5473-83.

76. Nonaka $\mathrm{T}$, et al. Prion-like properties of pathological TDP-43 aggregates from diseased brains. Cell Rep. 2013;4(1):124-34.

77. Brady OA, et al. Regulation of TDP-43 aggregation by phosphorylation and p62/SQSTM1. J Neurochem. 2011;116(2):248-59.
78. Li HY, et al. Hyperphosphorylation as a defense mechanism to reduce TDP-43 aggregation. PLoS One. 2011;6(8):e23075.

79. Mann JR, et al. RNA binding antagonizes neurotoxic phase transitions of TDP-43. Neuron. 2019;102(2):321$338 \mathrm{e} 8$.

80. Zhang P, et al. Chronic optogenetic induction of stress granules is cytotoxic and reveals the evolution of ALSFTD pathology. Elife. 2019;8:e39578.

81. McGurk L, et al. Poly(ADP-Ribose) prevents pathological phase separation of TDP-43 by promoting liquid demixing and stress granule localization. Mol Cell. 2018;71(5):703717 e9.

82. Liachko NF, Guthrie CR, Kraemer BC. Phosphorylation promotes neurotoxicity in a Caenorhabditis elegans model of TDP-43 proteinopathy. J Neurosci. 2010;30(48):16208-19.

83. Choksi DK, et al. TDP-43 Phosphorylation by casein kinase I $\varepsilon$ promotes oligomerization and enhances toxicity in vivo. Hum Mol Genet. 2014;23(4):1025-35.

Publisher's note Springer Nature remains neutral with regard to jurisdictional claims in published maps and institutional affiliations. 